\title{
Enhancement of Solubility and Dissolution Characteristics of Ibuprofen by Solid Dispersion Technique
}

\author{
Md. Abdullah Al Masum ${ }^{1}$, Florida Sharmin ${ }^{1}$, S. M. Ashraful Islam ${ }^{1}$ \\ and Md. Selim Reza ${ }^{2}$ \\ ${ }^{1}$ Department of Pharmacy, University of Asia Pacific, Dhanmondi, Dhaka-1209, Bangladesh \\ ${ }^{2}$ Department of Pharmaceutical Technology, Faculty of Pharmacy, University of Dhaka, \\ Dhaka-1000, Bangladesh
}

\begin{abstract}
In this study solid dispersions (SDs) of ibuprofen were prepared by melt dispersion technique using macrogol 4000 and macrogol 6000 as carrier. Physical mixtures (PMs) of ibuprofen were also prepared with the same carrier and in the same drug-carrier ratio $(1: 0.5,1: 1$ and 1:1.5) to compare the dissolution profile. The solid dispersions and physical mixtures were investigated for drug loading, saturation solubility and dissolution behavior. Saturation solubility study was carried out in phosphate buffer $(\mathrm{pH} 7.2), 0.1 \mathrm{~N} \mathrm{HCl}$ solution and distilled water. Solid dispersions were found effective to enhance the solubility of ibuprofen significantly in all the media. Dissolution test was carried out in two different media, phosphate buffer $(\mathrm{pH} 7.2)$ and $0.1 \mathrm{~N} \mathrm{HCl}$. Solid dispersion containing macrogol 6000 at the ratio of 1:1.5 (drug: carrier) showed faster and higher drug release and was found to be most effective among all the solid dispersions. Drug carrier interactions were studied by comparing Fourier Transform Infrared Spectroscopy (FT-IR) of solid dispersions with pure drug which revealed that the SDs were stable. So, solid dispersion may be an effective technique to enhance dissolution rate of ibuprofen.
\end{abstract}

Key words: Ibuprofen, macrogol, solid dispersion, saturation solubility, mean dissolution time.

\section{INTRODUCTION}

The poor dissolution characteristics of poorly water soluble drugs is a problem to the pharmaceutical industry because the dissolution rate of poorly water soluble drugs could be the rate limiting process in the absorption of a drug from a solid dosage form. With the recent advent of high throughput screening of potential therapeutic agents, the number of poorly soluble drug candidates has risen sharply and the formulation of poorly soluble compounds for oral delivery now a days presents one of the most frequent and greatest challenges to formulation scientists. ${ }^{1,2}$

Solubilization is the process by which the apparent solubility of a poorly water soluble drug is increased. Solubilization techniques include addition of a cosolvent, salt formation, prodrug design, complexation, particle size reduction and the use of surface active agents (micellization). ${ }^{3}$ Use of solvate

Correspondence to: Md. Selim Reza

Tel: +880-2-8612069; Fax: +880-2-8615583

Email: selim.du@gmail.com

Dhaka Univ. J. Pharm. Sci. 11(1): 1-6, 2012 (June) and hydrates, polymorphs, hydrotrophy, absorbents, $\mathrm{pH}$ adjustment, solubilizing vehicles, etc. are the some other physico-chemical approaches to enhancing oral absorption of poorly water soluble drugs. $^{4-6}$

Among the various approaches, the solid dispersion technique has been proved to be the most successful in improving the dissolution and bioavailability particularly of drugs having poor aqueous solubility. ${ }^{7}$ It has become popular because of its simplicity, cost effectiveness and advantages over other techniques. Solid dispersion is a mean of reducing particle size and the drug can be dispersed molecularly in amorphous particles (clusters) or in crystalline particles. ${ }^{8}$ It allows distribution of carrier component in between and around the drug and thus offers better surface characteristics and wetting.

Ibuprofen is an essential non-steroidal antiinflammatory drug characterized by poor aqueous solubility. Dissolution of ibuprofen is a rate limiting step that sometimes results in incomplete absorption 
due to poor dissolution and/or delayed dissolution. ${ }^{9}$ But rapid onset of action is vital for pain situations particularly in dental pain, rheumatoid and osteoarthritis and breast cancers. Moreover, ibuprofen has been rated as the safest conventional NSAID by spontaneous adverse drug reaction reporting systems in the UK. ${ }^{10}$ Again Ibuprofen is the most commonly used and most frequently prescribed NSAID. ${ }^{11}$ To improve solubility characteristics followed by dissolution of such drug is challenging and rational.

Solid dispersion of ibuprofen using different carrier has been reported. ${ }^{12,13}$ But in some cases higher amount of carrier was used which may be a problem to prepare tablets for human use. In this study we have used relatively less amount of carrier (macrogol 4000 and macrogol 6000 in 1:0.5, 1:1 and 1:1.5 ratio). In some cases drug dissolution was not increased as required. So an initiative has been taken to enhance the solubility and dissolution characteristics of ibuprofen by solid dispersion technique.

\section{MATERIALS AND METHODS}

Materials. Macrogol 6000 and Macrogol 4000 were purchased from Unichem Chemical Reagents. Ibuprofen was collected from ACI Pharmaceuticals Ltd, Dhaka, Bangladesh as a gift sample. All other ingredients were of analytical grade and collected from local market.

Preparation of solid dispersions. Solid dispersions were prepared by fusion method using macrogol 4000 and macrogol 6000 as carrier. The SDs were prepared at weight ratio of 1:0.5, 1:1, 1:1.5 (drug: carrier) and coded as $\mathrm{SD}(\mathrm{M} 4) 1 / 0.5$, $\mathrm{SD}(\mathrm{M} 4) 1 / 1$ and $\mathrm{SD}(\mathrm{M} 4) 1 / 1.5$, respectively for macrogol 4000 and $\mathrm{SD}(\mathrm{M} 6) 1 / 0.5, \mathrm{SD}(\mathrm{M} 6) 1 / 1$ and $\mathrm{SD}$ (M6)1/1.5, respectively for macrogol 6000 . The required amount of carrier and ibuprofen was melted in a beaker on a water bath maintained at $80^{\circ} \mathrm{C}$ and mixed thoroughly with a glass rod for $5 \mathrm{~min}$. The mixture was cooled rapidly by placing the beaker in an ice bath for 5 min to solidify, then powdered in a mortar, sieved through a 30-mesh screen and stored in a screw-cap vial at room temperature and stored in desiccators for further use.

Preparation of physical mixture. The physical mixtures (PMs) in the same weight ratio as the SDs mentioned above were prepared by thoroughly mixing the appropriate amounts of ibuprofen and carrier for $10 \mathrm{~min}$ in a mortar. The mixtures were coded as PM (M4) 1/0.5, PM (M4) 1/1 and PM (M4) $1 / 1.5$, respectively for macrogol 4000 and PM (M6) 1/0.5, PM (M6) 1/1 and PM (M6) 1/1.5, respectively for macrogol 6000. The mixtures were sieved through a 30-mesh sieve and stored in screw-cap vials at room temperature and used for further study.

Assay of samples. To assess the mixing uniformity of drug in the solid dispersions, ibuprofen standard sample and equivalent solid dispersion were dissolved in methanol separately. Methanol was chosen as the diluting solvent because ibuprofen is very soluble in methanol. ${ }^{14}$ The standard and sample solutions were suitably diluted by methanol and absorbance was measured by using a UV spectrophotometer (UV mini 1240, Shimadzu) at 221 $\mathrm{nm}$. The assay procedure was repeated thrice and standard deviation was calculated to see the uniformity.

Solubility studies. Solubility studies of pure Ibuprofen and solid dispersions were carried out in three different media. The media were $0.1 \mathrm{~N} \mathrm{HCl}$ solution, phosphate buffer ( $\mathrm{pH}$ 7.2) and distilled water. Standard ibuprofen, powder sample of solid dispersions equivalent to $100 \mathrm{mg}$ of pure ibuprofen were taken in $15 \mathrm{ml}$ screw cap test tubes containing $10 \mathrm{ml}$ of media. The solutions were shaken and kept aside for $24 \mathrm{hrs}$ with continuous stirring. After $24 \mathrm{hrs,}$ the sample solutions were filtered through Whatmann filter no. 1 and from the filtrate $1 \mathrm{ml}$ of solution was taken and diluted to a suitable concentration with respective media. The absorbance of the prepared dilutions was measured at $221 \mathrm{~nm}$ using UV-Visible spectrophotometer.

In vitro dissolution rate studies. The in vitro dissolution studies were carried out in USP XXI six stage dissolution rate test apparatus using $900 \mathrm{ml}$ of dissolution medium. Two dissolution media 
(phosphate buffer $\mathrm{pH} 7.2$ and $0.1 \mathrm{~N} \mathrm{HCl}$ solution) were used in this study. The temperature of the medium was maintained at $37 \pm 0.5^{\circ} \mathrm{C}$ throughout the experiment. The samples containing $100 \mathrm{mg}$ of ibuprofen or its equivalent in solid dispersions or physical mixtures were placed in the dissolution medium. Paddle was used at a stirring rate of $50 \mathrm{rpm}$. A $5 \mathrm{ml}$ aliquot was withdrawn at predetermined time intervals of at 5, 10, 20, 30, 45 and 60 minutes and then $5 \mathrm{ml}$ of fresh dissolution medium was replaced to maintain the constant volume of dissolution medium. From the samples collected, $1 \mathrm{ml}$ was diluted with dissolution medium and the absorbance of the diluted solutions were measured at $221 \mathrm{~nm}$ using Shimadzu UV-1201 UV/Visible double beam spectrophotometer (Shimadzu, Japan) against dissolution medium as blank. Percentage of drug release was calculated using the equation obtained from the standard curve prepared in each media.

To characterize the drug release rate in different experimental conditions, MDT (mean dissolution time), $\mathrm{T}_{50 \%}$ and $\mathrm{T}_{80 \%}$ dissolution efficiency (DE) were calculated from dissolution data according to the following equations ${ }^{15}$ :

$$
\begin{aligned}
& \mathrm{T}_{50 \%}=(0.5 / \mathrm{k})^{1 / \mathrm{n}} \\
& \mathrm{T}_{80 \%}=(0.8 / \mathrm{k})^{1 / \mathrm{n}} \\
& \mathrm{MDT}=(\mathrm{n} / \mathrm{n}+1) \cdot \mathrm{k}^{-1 / \mathrm{n}} \\
& D E=\frac{\int_{t_{1}}^{t_{2}} y \cdot d t}{y_{100} \times\left(t_{2}-t_{1}\right)} \times 100
\end{aligned}
$$

Where $\mathrm{n}$ is a release exponent and $\mathrm{y}$ is the percentage of dissolved product.

Mean dissolution time (MDT) was used to characterize the drug release rate from the dosage form. A higher value of MDT indicates a lower drug releasing ability of the solid dispersion and viceversa.

Drug-polymer interactions study. FT-IR spectra were taken in IR-Prestige 21, Shimadzu, Japan by scanning the sample in potassium bromide $(\mathrm{KBr})$ discs. Before taking the spectrum of the sample, a blank spectrum of air background was taken. The sample of pure drug, pure polymers and the solid dispersions containing both the drug and polymer were scanned over the frequency range 2000 $\mathrm{cm}^{-1}$ to $450 \mathrm{~cm}^{-1}$. The IR spectra of solid dispersions were compared with standard IR spectra of pure ibuprofen and respective carrier.

\section{RESULTS AND DISCUSSION}

The purpose of the study was to increase the dissolution rate of ibuprofen, a poorly water soluble drug. Macrogol 4000 and macrogol 6000 in different ratio were used to prepare SDs and PMs for this purpose. All the solid dispersions prepared by melting method were found to be granular, fine and free flowing. Assay results indicated that $98 \%-102 \%$ ibuprofen of theoretical drug loading was present in the solid dispersions.

Table 1 shows the saturated solubility of ibuprofen and solid dispersions in $0.1 \mathrm{~N} \mathrm{HCl}$ solution, phosphate buffer $\mathrm{pH} 7.2$ and distilled water. Saturation solubility study of solid dispersions using macrogol 4000 and macrogol 6000 were found effective in enhancing solubility in all the three media. Solid dispersions have been found to increase the solubility up to $8 \%$ in $0.1 \mathrm{~N} \mathrm{HCl}, 17 \%$ in

\begin{tabular}{|c|c|c|c|}
\hline \multirow{2}{*}{ Formulation } & $0.1 \mathrm{~N} \mathrm{HCl}$ & $\mathrm{pH} 7.2$ & Distilled water \\
\hline & \multicolumn{3}{|c|}{ Solubility $(\mathrm{mg} / \mathrm{ml}) \pm \mathrm{SD}(\mathrm{n}=3)$} \\
\hline SD(M4) $1 / 0.5$ & $0.061 \pm 0.011$ & $2.270 \pm 0.301$ & $0.112 \pm 0.025$ \\
\hline $\mathrm{SD}(\mathrm{M} 4) 1 / 1$ & $0.064 \pm 0.012$ & $3.090 \pm 0.211$ & $0.114 \pm 0.011$ \\
\hline $\mathrm{SD}(\mathrm{M} 4) 1 / 1.5$ & $0.064 \pm 0.012$ & $5.630 \pm 0.119$ & $0.197 \pm 0.031$ \\
\hline SD(M6) $1 / 0.5$ & $0.063 \pm 0.013$ & $3.890 \pm 0.103$ & $0.089 \pm 0.021$ \\
\hline $\mathrm{SD}$ (M6) $1 / 1$ & $0.066 \pm 0.011$ & $4.060 \pm 0.312$ & $0.086 \pm 0.024$ \\
\hline SD(M6) $1 / 1.5$ & $0.064 \pm 0.012$ & $4.010 \pm 0.218$ & $0.119 \pm 0.026$ \\
\hline Ibuprofen & $0.059 \pm 0.011$ & $3.410 \pm 0.311$ & $0.070 \pm 0.009$ \\
\hline
\end{tabular}
phosphate buffer ( $\mathrm{pH} 7.2$ ) and $70 \%$ in aqueous medium in comparison with pure ibuprofen.

Table 1. Saturated solubility of ibuprofen and solid dispersions in $0.1 \mathrm{~N} \mathrm{HCl}$, Phosphate buffer $\mathrm{pH} 7.2$ and distilled water. 
In vitro drug release in phosphate buffer ( $\mathrm{pH} 7.2)$ from solid dispersion and physical mixture prepared from macrogol 4000 and macrogol 6000 are shown in figures 1 and 2. Drug release from solid dispersions was found higher (up to $76.7 \%$ within $5 \mathrm{~min}$ ) than pure drug sample $(48.3 \%$ within $5 \mathrm{~min}$ ) from the beginning of dissolution study. But at this time point PM released relatively less amount of drug than the pure drug sample. Molecular dispersion and amorphous form of drug present in SD may be responsible for higher drug release. On the other hand presence of soluble carrier in the physical mixture may be a possible cause for initial lower drug release from physical mixture.

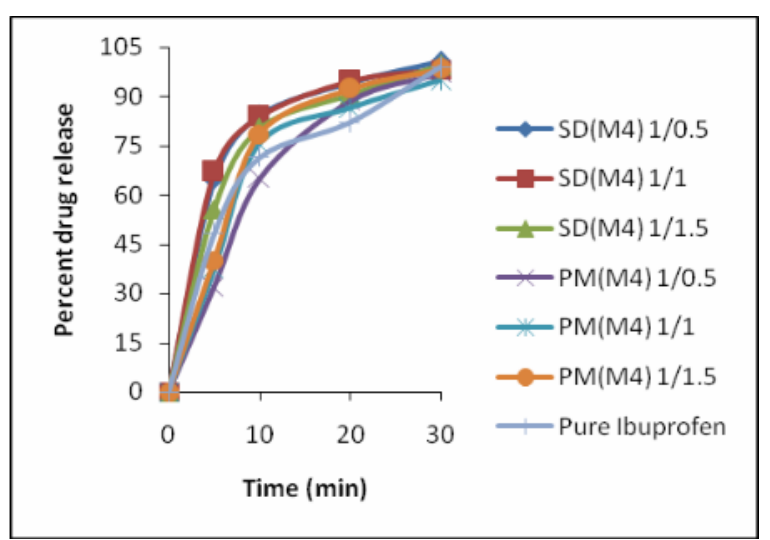

Figure 1. Percent drug release in phosphate buffer ( $\mathrm{pH}$ 7.2) from SD and PM containing macrogol 4000.

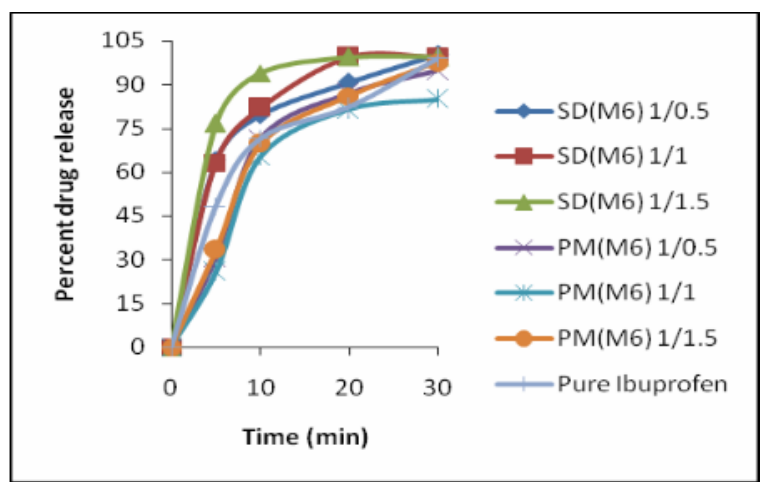

Figure 2. Percent drug release in phosphate buffer ( $\mathrm{pH}$ 7.2) from SD and PM containing Macrogol 6000.

However, drug release from physical mixtures increased rapidly after 5 min and 100\% drug was released within $45 \mathrm{~min}$. On the other hand, around $100 \%$ drug was released from solid dispersion within
20 minutes. So, solid dispersion may be an effective method to enhance dissolution of ibuprofen.

SD prepared from magrogol 6000 was found to release higher amount of drug ( $99 \%$ with in $20 \mathrm{~min}$ ) than SD prepared from magrogol 4000 (90\% within $20 \mathrm{~min}$ ). Again drug release was increased when amount of carrier was increased in the SD.

Dissolution studies were also done in $0.1 \mathrm{~N} \mathrm{HCl}$. Figure 3 and 4 show in vitro drug release in $0.1 \mathrm{~N}$ $\mathrm{HCl}$ from solid dispersions and physical mixtures prepared from macrogol 4000 and macrogol 6000. Drug release was very low in this medium as ibuprofen is practically insoluble in $0.1 \mathrm{~N} \mathrm{HCl}$ (Table 1). Only 5.89\% ibuprofen was dissolved within 1 hour from pure drug sample.

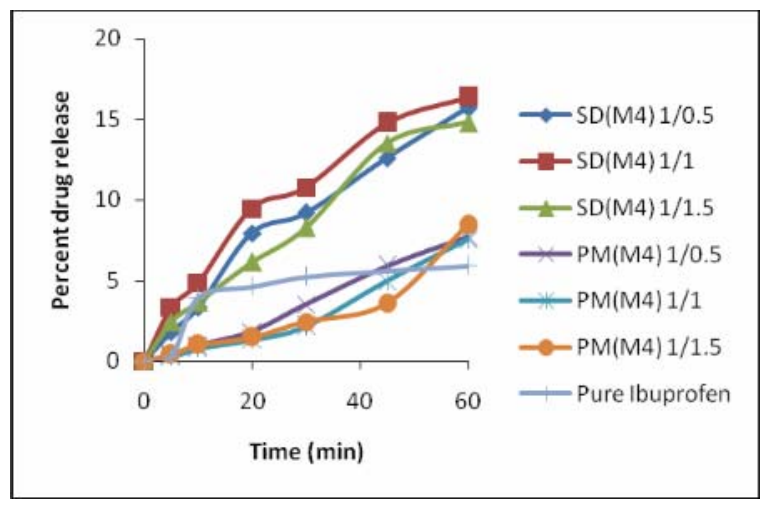

Figure 3. Percent drug release in $0.1 \mathrm{~N} \mathrm{HCl}$ from $\mathrm{SD}$ and $\mathrm{PM}$ containing macrogol 4000 .

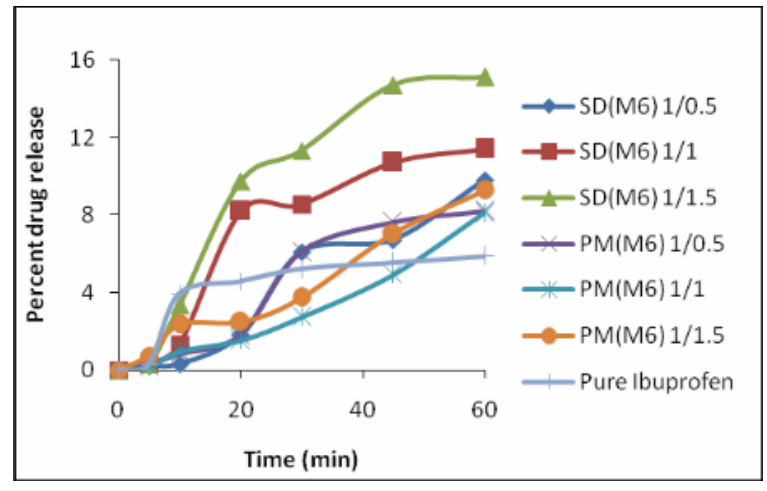

Figure 4. Percent drug release in $0.1 \mathrm{~N} \mathrm{HCl}$ from SD and PM prepared using macrogol 6000.

SD prepared from macrogol 4000 (Figure 3) was found to release $14.8 \%$ - $16.4 \%$ ibuprofen after 1 hour whereas the physical mixtures of the same carrier were found to release $7.6 \%-8.4 \%$ ibuprofen 
after 1 hour. Besides, solid dispersions prepared by using macrogol 6000 (Figure 4) were found to release $9.8 \%-15.1 \%$ after 1 hour and physical mixtures of the same carrier released $8.1-9.3 \%$ ibuprofen. Thus, solid dispersions and physical mixture prepared from both macrogol 4000 and macrogol 6000 can also improve ibuprofen release in acidic media.

MDT (mean dissolution time), $\mathrm{T}_{50 \%}$ (Time required for $50 \%$ drug release), $\mathrm{T}_{80 \%}$ (Time required for $80 \%$ drug release) and dissolution efficiency ( $\%$
DE) were calculated from dissolution data and presented in table 2. The MDT value of was found to be a function of carrier loading. Lower MDT of SD (M6) $1 / 1.5$ (2.84 min) indicates a higher dissolution rate of the formulation. Again, higher $\% \mathrm{DE}$ value of SD (M6) 1/1.5 (2.84 min) indicates the higher extend of drug release from the formulation. So both the rate and extent of drug release were apparently increased from solid dispersion.

Solubility $(\mathrm{mg} / \mathrm{ml}) \pm \mathrm{SD}(\mathrm{n}=3)$

Table 2, Successive fractional dissolution time $\left(\mathbf{T}_{50 \%}\right.$ and $\left.\mathrm{T}_{80 \%}\right)$, MDT (in $\min \pm \mathrm{SD}$ ) values and \% DE (20 min) of solid dispersions and physical mixtures.

\begin{tabular}{|c|c|c|c|c|}
\hline Formulation & MDT & $\mathrm{T}_{50 \%}$ & $\mathrm{~T}_{80 \%}$ & $\% \mathrm{DE}_{20 \mathrm{~min}}$ \\
\hline $\mathrm{SD}$ (M6) $1 / 0.5$ & $5.97 \pm 0.025$ & $1.30 \pm 0.025$ & $11.91 \pm 0.85$ & $80.72 \pm 2.50$ \\
\hline $\mathrm{SD}$ (M6) $1 / 1$ & $5.18 \pm 0.037$ & $1.26 \pm 0.035$ & $10.43 \pm 1.23$ & $84.69 \pm 3.40$ \\
\hline $\mathrm{SD}$ (M6) $1 / 1.5$ & $2.84 \pm 0.015$ & $0.15 \pm 0.01$ & $4.62 \pm 0.35$ & $92.80 \pm 2.15$ \\
\hline $\mathrm{PM}(\mathrm{M} 6)$ 1/0.5 & $14.55 \pm 0.62$ & $10.64 \pm 0.52$ & $28.15 \pm 1.97$ & $69.79 \pm 2.45$ \\
\hline $\mathrm{PM}(\mathrm{M} 6)$ 1/1 & $12.20 \pm 0.25$ & $8.18 \pm 0.18$ & $24.09 \pm 1.15$ & $64.08 \pm 2.25$ \\
\hline PM(M6) 1/1.5 & $11.74 \pm 0.93$ & $7.53 \pm 0.14$ & $23.38 \pm 2.12$ & $68.95 \pm 2.53$ \\
\hline $\mathrm{SD}(\mathrm{M} 4) 1 / 0.5$ & $5.68 \pm 0.57$ & $0.74 \pm 0.035$ & $10.69 \pm 1.47$ & $84.35 \pm 2.55$ \\
\hline $\mathrm{SD}(\mathrm{M} 4) 1 / 1$ & $5.65 \pm 0.68$ & $0.49 \pm 0.025$ & $10.01 \pm 0.87$ & $84.67 \pm 3.16$ \\
\hline $\mathrm{SD}(\mathrm{M} 4) 1 / 1.5$ & $7.30 \pm 0.75$ & $1.99 \pm 0.215$ & $14.84 \pm 1.22$ & $79.64 \pm 2.84$ \\
\hline PM(M4) 1/0.5 & $10.88 \pm 0.96$ & $5.99 \pm 0.57$ & $22.12 \pm 1.25$ & $67.41 \pm 1.55$ \\
\hline PM(M4) 1/1 & $12.22 \pm 1.01$ & $8.23 \pm 0.94$ & $24.12 \pm 1.20$ & $72.93 \pm 3.52$ \\
\hline PM(M4) 1/1.5 & $9.75 \pm 0.95$ & $4.95 \pm 0.14$ & $19.95 \pm 1.53$ & $76.67 \pm 2.53$ \\
\hline Pure Ibuprofen & $8.96 \pm 0.84$ & $3.79 \pm 0.18$ & $18.46 \pm 1.71$ & $71.14 \pm 2.55$ \\
\hline
\end{tabular}

IR spectra of pure Ibuprofen, carrier and SD are shown in figures 5-9. IR spectrum of pure ibuprofen (Figure 5) was identical with solid dispersions (Figures 8 \& 9). This indicates that there was no interaction between ibuprofen and carriers in the prepared solid dispersions. The spectra of solid dispersions were found to contain identical peaks of pure drug and carrier. In addition, no degradation of drug and carrier due to the high temperature during manufacturing was found from IR spectra.

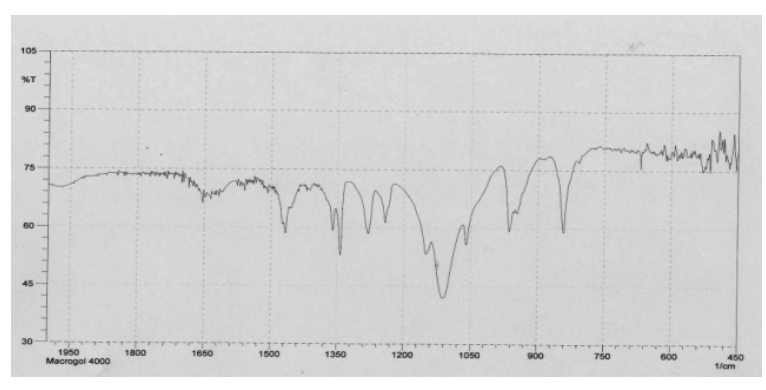

Figure 6. IR spectrum of macrogol 4000.

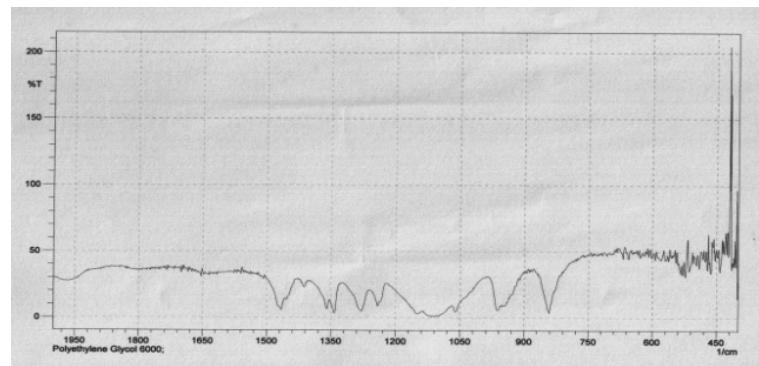

Figure 7. IR spectrum of macrogol 6000.

Figure 5. IR spectrum of ibuprofen. 


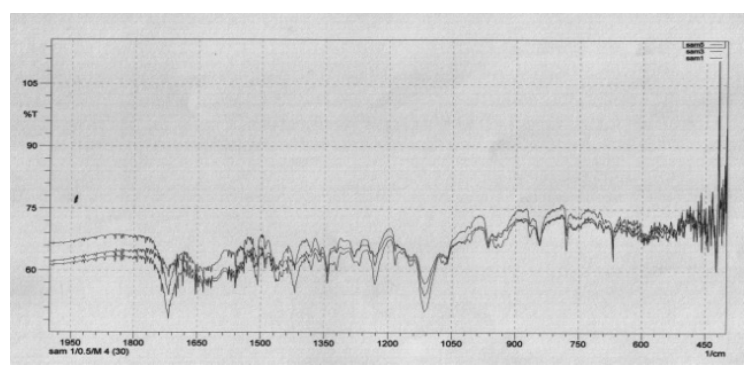

Figure 8. IR spectra of solid dispersions containing macrogol 4000 in different ratios.

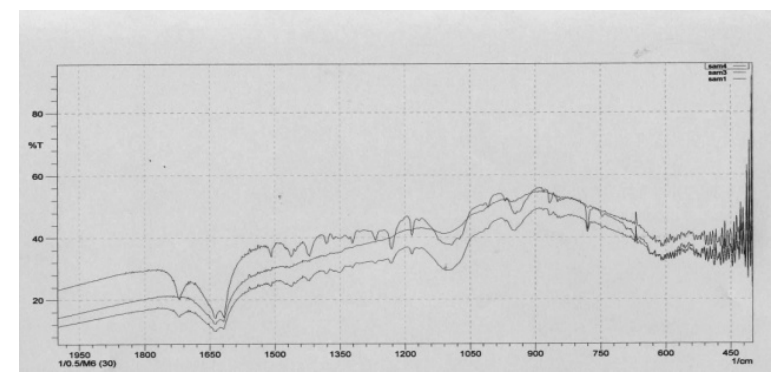

Figure 9. IR spectra of solid dispersions containing macrogol 6000 in different ratios.

\section{Conclusion}

Ibuprofen is practically insoluble in water and consequently its oral absorption is dissolution rate limited. Among the various approaches to improve the dissolution of poorly soluble drugs, the preparation of solid dispersions has often proven to be very successful for various drugs. ${ }^{7}$ All the prepared solid dispersions of ibuprofen using water soluble macrogols were found effective as compared to physical mixtures. Therefore, the application of solid dispersion technique for ibuprofen has been found promising for the further research and development of a suitable drug delivery system.

\section{References}

1. Patidar, K., Soni, M., Sharma, K.D. and Jain, K.S. 2010. Solid Dispersion: Approaches, Technology involved, Unmet need \& Challenges. Drug Invention Today. 2, 349-357
2. Leuner, C. and Dressman, J. 2000. Improving drug solubility for oral delivery using solid dispersions. Eur. J. Pharm. Biopharm. 50, 47-60.

3. James, S. and James, C.B. 2002. Encyclopedia of Pharmaceutical Technology. (2nd Edition), Marcel Dekker Inc., New York Basel, 2, pp. 1676-1685.

4. Satinder, A. and Stephen, S. 2001. Handbook of Modern Pharmaceutical Analysis, Academic Press, San Diego USA, London UK, Vol. 3, pp. 202-203.

5. Carter, S.J. 2002. Cooper and Gunn's Tutorial Pharmacy. (6th Edition), CBS Publishers and Distribotors, New Delhi, p. 13

6. Rasool, A.A., Hussain, A.A. and Dittert, L.W. 1991. Solubility enhancement of some water insoluble drugs in the presence of nicotinamide and related compounds. J. Pharm. Sci. 80, 387-393.

7. Ruchi, T., Gaurav T., Birendra, S. and Awani, K.R. 2009. Solid Dispersions: An Overview to Modify Bioavailability of Poorly Water Soluble Drugs. Int. J. PharmTech Res. 1, 13381349.

8. Pouton, C.W. 2000. Lipid formulations for oral administration of drugs: non-emulsifying, self-emulsifying and 'self-microemulsifying' drug delivery systems. Eur. $J$. Pharm. Sci. 2, 93-98

9. Mohammad, A.D. and Behzad, T. 2007. Investigation of solid dispersion technique in improvement of physicochemical characteristics of ibuprofen powder. Iranian J. Pharm. Sci. 3, 69-76.

10. Rabia, B. and Nousheen, A. 2010. An overview of clinical pharmacology of ibuprofen. Oman Med. J. 25, 155-161

11. Tripathi, K.D. 2003. Non steroidal anti inflammatory drugs and anti pyretic analgesics. Essentials of medical pharmacology. (5th ed.), Jaypee Brothers, New Delhi, p. 176.

12. Tapan, K.G., Hemant, B., Amit, A. and Dulal, K.T. 2010. Solubility enhancement of ibuprofen in the presence of hydrophilic polymer and surfactant. Int. J. Appl. Biol. Pharm. Tech. 1, 793-800

13. Khan, G.M. and Jiabi, Z. 1998. Preparation, characterization and dissolution studies of ibuprofen solid dispersion using polyethylene glycol, talc and PEG-talk as dispersion carriers. Drug Dev. Ind. Pharm. 24, 455-462.

14. USP 32-NF 27. 2009. The United States pharmacopeia - The National Formulary. Rockville, MD: The United States Pharmacopeial Convention, Inc.

15. Mockel, J.E. and Lippold, B.C. 1993. Zero order release from hydrocolloid matrices. Pharm. Res. 10, 1066-1070. 